Georgian Mathematical Journal

Volume 11 (2004), Number 4, 753-758

\title{
ON GENERALIZED STEP-FUNCTIONS AND SUPERPOSITION OPERATORS
}

\author{
A. KHARAZISHVILI
}

\begin{abstract}
For a given $\sigma$-ideal of sets, the notion of a generalized stepfunction is introduced and investigated in connection with the problem of sup-measurability of certain functions of two variables, regarded as superposition operators.
\end{abstract}

2000 Mathematics Subject Classification: 28A05, 28D05.

Key words and phrases: generalized step-function, superposition operator, Lebesgue measure, Baire property, $\sigma$-ideal of sets, base of a $\sigma$-ideal.

Let $R$ denote the real line and let $\Phi: R \times R \rightarrow R$ be a function of two variables. Then this $\Phi$ can be treated as a superposition operator defined as follows: for any function $f: R \rightarrow R$, we put

$$
(\Phi(f))(x)=\Phi(x, f(x)) \quad(x \in R) .
$$

Sometimes, $\Phi$ is also called a Nemytskii superposition operator.

Let $\lambda$ denote the standard Lebesgue measure on $R$. In many cases, it is important to know whether a given superposition operator $\Phi$ preserves the class $L(R)$ of all real-valued Lebesgue measurable functions on $R$ (i.e., $\Phi(f)$ is $\lambda$ measurable whenever $f$ is $\lambda$-measurable). There are various sufficient conditions under which $\Phi$ maps $L(R)$ into itself.

In particular, if $\Phi$ is $\lambda$-measurable with respect to the first variable and continuous with respect to the second variable (the so-called Carathéodory classical conditions), then $\Phi$ preserves $L(R)$ or, in short, $\Phi$ is sup-measurable. In such a case, $\Phi$ is also $\lambda_{2}$-measurable where $\lambda_{2}=\lambda \times \lambda$ stands for the two-dimensional Lebesgue measure on the plane $R^{2}$.

Other conditions for the sup-measurability of $\Phi$ can be found, e.g., in [1].

Under some additional set-theoretical axioms, there exist sup-measurable $\Phi$ which are not $\lambda_{2}$-measurable (see, for instance, [2], [3], [4], [5]). On the other hand, as Shelah and Roslanowski have recently announced, the statement "all sup-measurable operators $\Phi$ are $\lambda_{2}$-measurable" is consistent with ZFC theory.

In this paper, we are focused on the following problem: give a characterization of all those functions $f \in L(R)$ for which there exists a superposition operator $\Phi$ having rather good descriptive properties and such that $\Phi(f)$ does not belong to $L(R)$. In order to solve this problem, we need several auxiliary notions and propositions. First of all let us recall the following classical statement from descriptive set theory.

Lemma 1. Let $E$ be a Polish topological space, $E^{\prime}$ be a metric space, and let $h: E \rightarrow E^{\prime}$ be a continuous mapping whose range $\operatorname{ran}(h)$ is uncountable. Then 
there exists a set $C \subset E$ homeomorphic to the Cantor discontinuum such that the restriction $h \mid C$ is injective (hence $h \mid C$ is a homeomorphism between $C$ and $h(C))$.

For the proof of this lemma see, e.g., [6].

Recall that $f \in L(R)$ is a step-function if $\operatorname{card}(\operatorname{ran}(f)) \leq \omega$, i.e., the range of $f$ is at most countable. We shall say that $f \in L(R)$ is a generalized stepfunction if there exists at least one step-function $g \in L(R)$ such that $f$ and $g$ are equivalent (with respect to the measure $\lambda$ ).

Lemma 2. If $f \in L(R)$, then the following two assertions are equivalent:

1) $f$ is not a generalized step-function;

2) there exists a set $Y \subset R$ with $\lambda^{*}(Y)>0$ such that the restriction $f \mid Y$ is injective.

Proof. The implication 2) $\Rightarrow 1$ ) is trivial. Let us prove the implication 1 ) $\Rightarrow 2$ ). Suppose that $f \in L(R)$ satisfies 1$)$. Let us denote

$$
T_{0}=\left\{t \in \operatorname{ran}(f): \lambda\left(f^{-1}(t)\right)>0\right\} .
$$

Evidently, we have card $\left(T_{0}\right) \leq \omega$. Since $f$ is not a generalized step-function, we also have $\lambda\left(R \backslash f^{-1}\left(T_{0}\right)\right)>0$. Moreover, applying the classical Luzin theorem to $f$, we claim that there exists a closed set $P \subset R \backslash f^{-1}\left(T_{0}\right)$ with $\lambda(P)>0$ for which the restriction $f \mid P$ is continuous and $\operatorname{card}(\operatorname{ran}(f \mid P))>\omega$.

Let us put $h=f \mid P$ and $T=\operatorname{ran}(h)$. Then $\lambda\left(h^{-1}(t)\right)=0$ for each $t \in T$. Denote by $\alpha$ the least ordinal number of cardinality continuum and let $\left(P_{\xi}\right)_{\xi<\alpha}$ be an injective family of all closed subsets of $P$ having strictly positive $\lambda$ measure. Construct, by using the method of transfinite recursion, a family $\left\{y_{\xi}: \xi<\alpha\right\}$ of points of $P$. Namely, take an ordinal $\xi<\alpha$ and suppose that the partial family $\left\{y_{\zeta}: \zeta<\xi\right\}$ has already been defined. Keeping in mind Lemma 1 , it is not difficult to check that

$$
P_{\xi} \backslash \cup\left\{h^{-1}\left(h\left(y_{\zeta}\right)\right): \zeta<\xi\right\} \neq \varnothing .
$$

Hence there exists a point $y$ belonging to $P_{\xi} \backslash \cup\left\{h^{-1}\left(h\left(y_{\zeta}\right)\right): \zeta<\xi\right\}$.

We put $y_{\xi}=y$. By proceeding in the same manner as above, the required family of points $\left\{y_{\xi}: \xi<\alpha\right\}$ will be constructed. Denote now $Y=\left\{y_{\xi}: \xi<\alpha\right\}$. It follows directly from our construction that $Y$ is a partial selector of the disjoint family of sets $\left\{h^{-1}(t): t \in T\right\}$. This implies that the restriction $h \mid Y$ (consequently, the restriction $f \mid Y$ ) is injective. Moreover, since $P_{\xi} \cap Y \neq \varnothing$ for each $\xi<\alpha$, we easily infer that $\lambda^{*}(Y)=\lambda(P)>0$. This completes the proof of Lemma 2.

Lemma 3. If $f \in L(R)$ is not a generalized step-function, then there exists a $\lambda$-nonmeasurable set $X \subset R$ for which the restriction $f \mid X$ is injective.

Proof. According to Lemma 2, there exists a set $Y \subset R$ with $\lambda^{*}(Y)>0$ such that $f \mid Y$ is an injection. If $Y$ is not measurable in the Lebesgue sense, then we are done. Suppose now that $Y \in \operatorname{dom}(\lambda)$ and hence $\lambda(Y)>0$. It is well known (see, e.g., [7] or [8]) that $Y$ contains a nonmeasurable subset with respect to $\lambda$. 
Take any such subset and denote it by $X$. Clearly, $f \mid X$ is an injection and the proof is completed.

Theorem 1. Let $f \in L(R)$ and suppose that $f$ is not a generalized stepfunction. Then there exists a superposition operator $\Phi: R \times R \rightarrow R$ satisfying the following relations:

1) $\operatorname{ran}(\Phi)=\{0,1\}$;

2) for any $x \in R$, the partial function $\Phi(x, \cdot)$ is lower semi-continuous;

3) for any $y \in R$, the partial function $\Phi(\cdot, y)$ is lower semi-continuous;

4) $\Phi$ is a $\lambda_{2}$-measurable operator;

5) the function $\Phi(f)$ is not $\lambda$-measurable.

Proof. According to Lemma 3, there exists a $\lambda$-nonmeasurable set $X \subset R$ for which the restriction $f \mid X$ is injective. Define the required superposition operator $\Phi$ as follows:

$$
\begin{array}{ll}
\Phi(x, y)=0 & (x \in X, y=f(x)) \\
\Phi(x, y)=1 & (x \in R \backslash X, \quad y=f(x)), \\
\Phi(x, y)=1 & (x \in R, y \in R, \quad y \neq f(x)) .
\end{array}
$$

For this $\Phi$, relations 1), 2) and 3) are verified directly. Further, since the graph of $f$ is a $\lambda_{2}$-measure zero subset of $R^{2}$, we claim that $\Phi$ is equivalent to 1 and, consequently, $\Phi$ is $\lambda_{2}$-measurable. Finally, we have

$$
\Phi(x, y)=0 \Leftrightarrow(x \in X \& y=f(x))
$$

whence it follows that $(\Phi(f))^{-1}(0)=X$ and therefore $\Phi(f)$ is not $\lambda$-measurable. This ends the proof.

Theorem 2. Let $f \in L(R)$ and suppose that $f$ is not a generalized stepfunction. Then there exists a superposition operator $\Psi: R \times R \rightarrow R$ such that:

1) $\operatorname{ran}(\Psi)=\{1,2\}$;

2) for any $x \in R$, the partial function $\Psi(x, \cdot)$ is lower semi-continuous;

3) for any $y \in R$, the partial function $\Psi(\cdot, y)$ is lower semi-continuous;

4) $\Psi$ is a $\lambda_{2}$-nonmeasurable operator;

5) the function $\Psi(f)$ is $\lambda$-nonmeasurable.

Proof. By using the method of transfinite recursion and applying the standard argument (cf. [9]), we can define an injective function $g: R \rightarrow R$ whose graph is $\lambda_{2}$-thick in $R^{2}$ and does not intersect the graph of $f$. Let $\Phi_{g}$ denote the characteristic function of the graph of $g$ (regarded as a subset of $R^{2}$ ). We put

$$
\Psi=\Phi+1-\Phi_{g}
$$

where $\Phi$ is the superposition operator of Theorem 1. It is easy to verify that $\Psi$ is the required superposition operator, i.e., $\Psi$ satisfies all the relations 1$)-5$ ) above.

Remark 1. If a superposition operator $\Phi: R \times R \rightarrow R$ is $\lambda$-measurable with respect to the first variable, then $\Phi(f)$ is $\lambda$-measurable for every generalized 
step-function $f \in L(R)$. We thus see (in view of Theorem 1 ) that the generalized step-functions are exactly those functions $f \in L(R)$ for which any superposition operator $\Phi$ measurable with respect to the first variable yields measurable $\Phi(f)$.

Remark 2. If a superposition operator $\Phi: R \times R \rightarrow R$ is continuous with respect to the first variable and lower semi-continuous (more generally, Borel) with respect to the second variable, then $\Phi$ is a Borel mapping from $R^{2}$ into $R$, hence $\Phi$ is also sup-measurable.

Remark 3. Theorems 1 and 2 admit direct analogues for functions possessing the Baire property (detailed information about this property can be found in [6] and [8]). These analogues can be proved by the same scheme as for Lebesgue measurable functions. Only one essential moment should be mentioned. Namely, the proofs of Theorems 1 and 2 are based on the classical Luzin theorem concerning the structure of $\lambda$-measurable functions. Since we cannot apply the Luzin theorem to functions possessing the Baire property, we must replace this theorem by an appropriate similar statement. Such a statement is well known in general topology (see [6]) and is formulated as follows.

Let $E_{1}$ be a topological space, $E_{2}$ be a topological space with a countable base and let $f: E_{1} \rightarrow E_{2}$ be a mapping possessing the Baire property. Then there exists a first category set $Z \subset E_{1}$ such that the restriction $f \mid\left(E_{1} \backslash Z\right)$ is continuous.

We may assume, without loss of generality, that $Z$ is an $F_{\sigma}$-subset of $E_{1}$, hence $E_{1} \backslash Z$ is a $G_{\delta^{-}}$set in $E_{1}$. If the original space $E_{1}$ is Polish, then $E_{1} \backslash Z$ is also Polish (by virtue of the Alexandrov theorem). Consequently, if $E_{1}$ is a Polish space and $E_{2}=R$, we are able to apply Lemma 1 to the continuous function $f \mid\left(E_{1} \backslash Z\right)$.

Under some additional set-theoretical axioms, Lemma 2 admits a significant generalization. Let us consider some abstract version of this lemma.

Fix an uncountable set $E$ and a proper $\sigma$-ideal $\mathcal{I}$ of subsets of $E$, containing all singletons in $E$. We shall say that $g: E \rightarrow R$ is a step-function if $\operatorname{card}(\operatorname{ran}(g)) \leq$ $\omega$. We shall say that $f: E \rightarrow R$ is a generalized step-function with respect to $\mathcal{I}$ if there exists at least one step-function $g: E \rightarrow R$ for which we have

$$
\{x \in E: f(x) \neq g(x)\} \in \mathcal{I},
$$

i.e., $f$ and $g$ are $\mathcal{I}$-equivalent functions. Recall that a family of sets $\mathcal{B} \subset \mathcal{I}$ forms a base of $\mathcal{I}$ if, for any set $Y \in \mathcal{I}$, there exists a set $Z \in \mathcal{B}$ such that $Y \subset Z$.

The following statement is valid.

Theorem 3. Let $\operatorname{card}(E)=\omega_{1}$, let $\mathcal{I}$ be a proper $\sigma$-ideal of subsets of $E$, containing all singletons in $E$ and possessing a base whose cardinality does not exceed $\omega_{1}$, and let $f: E \rightarrow R$ be a function. Then the following two assertions are equivalent:

1) $f$ is not a generalized step-function with respect to $\mathcal{I}$;

2) there exists a set $X \subset E$ such that $X \notin \mathcal{I}$ and the restriction $f \mid X$ is injective. 
Proof. The implication 2$) \Rightarrow 1$ ) is evident. Let us establish the validity of the implication 1) $\Rightarrow 2$ ). Suppose that $f$ satisfies 1) and introduce the following two sets:

$$
T_{0}=\left\{t \in \operatorname{ran}(f): f^{-1}(t) \notin \mathcal{I}\right\}, \quad T_{1}=\left\{t \in \operatorname{ran}(f): f^{-1}(t) \in \mathcal{I}\right\} .
$$

According to our assumption, there exists a base $\left\{B_{\xi}: \xi<\omega_{1}\right\}$ of the given $\sigma$-ideal $\mathcal{I}$. Only two cases are possible.

1. $\operatorname{card}\left(T_{0}\right)=\omega_{1}$. In this case we may write $T_{0}=\left\{t_{\xi}: \xi<\omega_{1}\right\}$ where $t_{\xi} \neq t_{\zeta}$ for all $\xi<\omega_{1}, \zeta<\omega_{1}, \xi \neq \zeta$. Consider the family of sets $\left\{f^{-1}\left(t_{\xi}\right) \backslash B_{\xi}: \xi<\omega_{1}\right\}$. Obviously, $f^{-1}\left(t_{\xi}\right) \backslash B_{\xi} \neq \varnothing$ for each ordinal $\xi<\omega_{1}$. Let $x_{\xi} \in f^{-1}\left(t_{\xi}\right) \backslash B_{\xi}$ for any $\xi<\omega_{1}$, and let $X=\left\{x_{\xi}: \xi<\omega_{1}\right\}$. From the definition of $X$ it immediately follows that the restriction $f \mid X$ is an injection. Moreover, we have $X \backslash B_{\xi} \neq \varnothing$ whenever $\xi<\omega_{1}$. The latter circumstance implies at once that the set $X$ does not belong to $\mathcal{I}$.

2. $\operatorname{card}\left(T_{0}\right) \leq \omega$. In this case we obtain $\operatorname{card}\left(T_{1}\right)=\omega_{1}$ and $f^{-1}\left(T_{1}\right) \notin \mathcal{I}$ (since our $f$ is not a generalized step-function with respect to $\mathcal{I}$ ). Let us construct, by using the method of transfinite recursion, an $\omega_{1}$-sequence $\left\{x_{\xi}: \xi<\omega_{1}\right\}$ of points of $f^{-1}\left(T_{1}\right)$. Suppose that, for an ordinal $\xi<\omega_{1}$, the partial family of points $\left\{x_{\zeta}: \zeta<\xi\right\}$ has already been defined. Clearly, the set $\left(\cup\left\{f^{-1}\left(f\left(x_{\zeta}\right)\right)\right.\right.$ : $\zeta<\xi\}) \cup B_{\xi}$ belongs to $\mathcal{I}$. Therefore,

$$
f^{-1}\left(T_{1}\right) \backslash\left(\left(\cup\left\{f^{-1}\left(f\left(x_{\zeta}\right)\right): \zeta<\xi\right\}\right) \cup B_{\xi}\right) \neq \varnothing .
$$

Choose any element $x$ from the above nonempty set and put $x_{\xi}=x$. Proceeding in this manner, we are able to construct the required $\omega_{1}$-sequence $\left\{x_{\xi}: \xi<\omega_{1}\right\}$. Finally, put $X=\left\{x_{\xi}: \xi<\omega_{1}\right\}$. In view of our construction, $X$ is a partial selector of the disjoint family of sets $\left\{f^{-1}(t): t \in T_{1}\right\}$. Hence the restriction of $f$ to $X$ is injective. Furthermore, $X \backslash B_{\xi} \neq \varnothing$ for all ordinals $\xi<\omega_{1}$, whence it follows that $X$ does not belong to $\mathcal{I}$.

Remark 4. Assume the Continuum Hypothesis $(\mathrm{CH})$ and take as $\mathcal{I}$ the $\sigma$ ideal $\mathcal{L}$ of all Lebesgue measure zero subsets of $R$. Let $f: R \rightarrow R$ be a function distinct from all generalized step-functions with respect to $\mathcal{L}$. Suppose also that the graph of $f$ is a set of $\lambda_{2}$-measure zero. Then it is not difficult to show that, for such an $f$, there always exists a superposition operator $\Phi: R \times R \rightarrow R$ satisfying the relations 1)-5) of Theorem 1 . In this connection, let us emphasize that our $f$ does not need to be a $\lambda$-measurable function.

Remark 5. Let $E$ be a set, $\mathcal{I}$ be a proper $\sigma$-ideal of subsets of $E$ and let $\mathcal{S}$ be a $\sigma$-algebra of subsets of $E$ such that $\mathcal{I} \subset \mathcal{S}$. Elements of $\mathcal{S}$ are usually called measurable sets in $E$ and elements of $\mathcal{I}$ are called negligible sets in $E$. The triple $(E, \mathcal{S}, \mathcal{I})$ is called a measurable space with negligibles (see, e.g., [10]). If $X \subset E$ and $X \notin \mathcal{I}$, then, in general, we cannot assert that $X$ contains at least one subset which does not belong to $\mathcal{S}$. However, in some situations the specific features of a given $\sigma$-ideal $\mathcal{I}$ imply that any nonnegligible set in $E$ contains a nonmeasurable subset. For example, assume again that $\operatorname{card}(E)=\omega_{1}$ and that $E$ is a topological space of second category, whose all singletons are of first category. Let $\mathcal{I}=\mathcal{K}(E)$ denote the $\sigma$-ideal of all first category subsets of $E$ and 
suppose that this $\sigma$-ideal possesses a base whose cardinality does not exceed $\omega_{1}$. Denote also by $\mathcal{B}(E)$ the $\sigma$-algebra of all subsets of $E$ having the Baire property (obviously, $\mathcal{K}(E) \subset \mathcal{B}(E)$ ). Then, for any set $X \subset E$, the following two assertions are equivalent:

(a) $X \notin \mathcal{K}(E)$ (i.e., $X$ is not of first category in $E$ );

(b) there exists a set $Y \subset X$ such that $Y \notin \mathcal{B}(E)$ (i.e., $Y$ does not have the Baire property in $E$ ).

The proof of the equivalence of (a) and (b) can be found in [11] where some related results are also presented. Notice once more that this equivalence rests only on the inner properties of the $\sigma$-ideal $\mathcal{K}(E)$ and does not touch upon the structure of the $\sigma$-algebra $\mathcal{B}(E)$.

\section{REFERENCES}

1. I. V. Shragin, Conditions for the measurability of superpositions. (Russian) Dokl. Akad. Nauk SSSR 197(1971), 295-298.

2. Z. Grande and J. Lipiński, Un exemple d'une fonction sup-mesurable qui n'est pas mesurable. Colloq. Math. 39(1978), No. 1, 77-79.

3. A. B. Kharazishvili, Some questions of the theory of invariant measures. (Russian) Soobshch. Akad. Nauk Gruzin. SSR 100(1980), No. 3, 533-536.

4. M. Balcerzak, Some remarks on sup-measurability. Real Anal. Exchange 17(1991/92), No. 2, 597-607.

5. M. A. KrasnoselskiI and A.V. PokrovskiI , Systems with hysteresis. (Translation from Russian) Springer-Verlag, Berlin, 1988.

6. K. Kuratowski, Topology. I. Academic Press, New York-London; Państwowe Wydawnictwo Naukowe, Warsaw, 1966.

7. I. P. Natanson, Theory of functions of real variable. (Russian) Second edition, revised. Gosudarstv. Izdat. Tehn.-Teoret. Lit., Moscow, 1957.

8. J. C. Охтовy, Measure and category. A survey of the analogies between topological and measure spaces. Graduate Texts in Mathematics, 2. Springer-Verlag, New York-Berlin, 1971.

9. A. B. Kharazishvili, Strange functions in real analysis. Marcel Dekker, Inc., New YorkBasel, 2000.

10. D. H. Fremlin, Measure-additive coverings and measurable selectors. Dissertationes Math. (Rozprawy Mat.) 260(1987), 116 pp.

11. A. B. Kharazishvili, The Baire property and its applications. (Russian) Tbiliss. Gos. Univ. Inst. Prikl. Mat. Trudy 43(1992), 117 pp.

(Received 30.08.2003)

Author's Address:

Alexander Kharazishvili

I. Vekua Institute of Applied Mathematics

I. Javakhishvili Tbilisi State University

2, University St., Tbilisi 0143, Georgia

E-mail: kharaz@saba.edu.ge 